\title{
El «Vocabulario» de El dialecto vulgar salmantino, de José Lamano y Beneite: Análisis macro y microestructural*
}

\author{
M. ${ }^{a}$ MONTSERRAT MURIANO RODRÍGUEZ \\ Universidade da Coruña
}

\section{INTRODUCCIÓN}

Varios han sido los trabajos que he dedicado a poner de manifiesto la relevancia del Vocabulario de José de Lamano y Beneite para la incorporación de acepciones con marcación dialectal salmantina en la décima quinta edición del $D R A E^{1}$. No obstante, en esta ocasión mi aportación se centrará exclusivamente en un asunto todavía inexplorado: el análisis de los aspectos macro y microestructurales más relevantes de la tercera parte de la obra del canónigo salmantino, tarea esta que nos acerca a la concepción lexicográfica de la obra por parte de este aficionado a la dialectología parte de cuyo lemario ha pasado a engrosar las páginas del Diccionario académico.

Una buena manera de comenzar el análisis de esta tercera parte de El dialecto vulgar salmantino es la reproducción de las palabras del insigne dialectólogo salmantino don Antonio Llorente quien, en el estudio introductorio a la edición facsímil de la obra, indica que:

El Vocabulario [...] es, con mucho, lo más importante, más sugestivo, lo más aprovechable, incluso podríamos decir lo más científico del libro del canónigo salmantino, y, por sí solo, este repertorio del léxico provincial se habría merecido con creces el premio que la Real Academia Española concedió en su día a la obra de Lamano (Lamano y Beneite 1915: V) ${ }^{2}$.

${ }^{*}$ Este trabajo se inscribe en los proyectos de investigación Diccionario del español de la Edad de Plata (continuación) (FFI2011-23085/FILO, MINECO) y Léxico de la vida cotidiana (10PXIB104235PR, Xunta de Galicia), cuyo investigador principal es el profesor Pérez Pascual. Asimismo, esta contribución ha sido financiada en el marco de la Red de Lexicografia (Relex, Ref. CN2012/290), subvencionada por la Consellería de Cultura, Educación e Ordenación, Xunta de Galicia, cuya coordinadora es la profesora Sánchez Palomino.

${ }^{1}$ Vid. Muriano Rodríguez (1997-1998; 2001; 2002a y 2002b); vid. especialmente Muriano Rodríguez (2012: 446-450) donde analizo el lemario incluido en el repertorio de Lamano en base a una clasificación de dicho material, fundamentalmente, como arcaísmos léxicos, variantes fonéticas, léxico general y salmantinismos.

${ }^{2} \mathrm{Si}$ bien este juicio es de 2002, ya en su tesis doctoral sobre el habla de la Ribera, no duda en indicar que la obra «representa el mayor esfuerzo hecho hasta ahora para estudiar el habla salmantina en su totalidad geográfica, así como para compilar su rico vocabulario» (Llorente Maldonado, 1947: 42). Y continúa: «Es un trabajo admirable, por sus ambiciones, por comprender a todas las regiones salmantinas, por recoger la mayoría de los vocablos regionales». No obstante, también pone de manifiesto las carencias de este clérigo, aprendiz de dialectólogo: «pero tiene el fallo de su método anticuado e impropio, de su rara terminología y de la muchas veces errónea explicación de los fenómenos, cosa, al fin, natural en un autor que no seguía la moderna escuela lingüística, sino que se guiaba por el antiguo y tradicional método del Seminario». Recordemos que dichas carencias, en especial el «no hallar esta memoria al corriente de la bibliografía del asunto que trata» ( $A R A E$, legajo 251, expediente 1) son las que motivaron que únicamente obtuviese el accésit del premio de la Academia al que presentó El dialecto vulgar salmantino (sobre este asunto vid. Muriano Rodríguez $2002 \mathrm{~b}$ ). 


\section{LA MACROESTRUCTURA DEL VOCABULARIO}

Consta de 5243 entradas, aunque, según Llorente «el Vocabulario de D. José de Lamano, uno de los léxicos regionales y provinciales más ricos, consta de 5.237 entradas y, en total, de 6.427 acepciones» (Sánchez León, 1995: 12). En mi recuento manual he localizado 6101 acepciones directas y 598 remisiones a otra entrada que puede constar, a su vez, de una o varias acepciones, por lo que el recuento exacto de acepciones se haría interminable $y$, en realidad, no aporta información imprescindible ${ }^{3}$.

Lo realmente significativo es que, de esas 598 remisiones de las que he hablado, 501 son entradas que constan únicamente de la remisión ${ }^{4}$ o incluyen algún comentario sobre localización geográfica, nivel de uso con respecto a la entrada a la que remite o, simplemente, un ejemplo 5 .

El vocabulario contempla, pues, varias posibilidades de presentación de la información en estos «artículos remisivos». La clasificación que he realizado está basada en los siguientes aspectos:

1. Artículos que únicamente incluyen un 'V.' que remite a otro lema del Vocabulario:

Esborcillar, a. V. Esborcellar.

Vafear, n. V. Vadear.

Valona, f. V. Baluga.

Verdulago, m. V. Verdolaga.

Vilesa, f. V. Vilera.

Yerbera, f. Empina.

2. Artículos que incluyen información sobre la localización geográfica de la voz.

Serondo, da, adj. V. Ceriondo. (Ribera del Duero).

Vajear, int. V. Vadear. (Sierra de Francia).

Verrondio, ia, adv. V. Verreondo. (Armuña).

3. Artículos que incluyen información sobre el uso de la voz y de aquella a la que remite:

Apazconar, a. V. Apasconar. Úsanse entrambos términos indistintamente.

Escaldunciar, a. V. Escalduciar. Úsanse promiscuamente ambos términos.

Moflear, a. V. Mofear . Úsanse promiscuamente ambos términos, si bien este último se emplea con más frecuencia, quizás por ser más expresivo, ya que indica la burla que se hace con los mofletes.

Sojadro, m. V. Sojado. Úsanse promiscuamente, en dialecto, ambos vocablos.

4. Artículos que incluyen algún ejemplo:

Arribe, m. V. Arribadero:

\footnotetext{
${ }^{3}$ En contadas ocasiones indica exactamente a qué acepción remite. Es el caso de acuellar donde dice 'acollar, en la acepción última', churro 'churra, en la última acepción' o liño 'linio, en la segunda acepción'.

${ }^{4}$ Representan el 9,55\% de la macroestructura del vocabulario.

${ }^{5}$ En el Anexo reproduzco todas estas entradas por orden alfabético.
} 
«... y en último término, como telón de fondo, los azulados arribes de la orilla opuesta, en cuya oscura silueta de destaca sobre un cielo transparente»

(Maldonado, Del campo, pág. 116).

Escarrapicharse, r. V. Escarramancharse:

«Diz que iba escarrapichado

el mamarracho del indio

sobre el pobre animalejo

con su garrocha y culeto»

(Torres Villarroel, Obras, T. VII).

5. Artículos que contienen información de varios de los puntos anteriores:

Abaivus. V. Abar. Forma usual en la Ribera del Duero.

«Abaivus di ahi, que no vus quiero ver endelante de mí».

Escaer, int. V. Escaecer. Emplean este vocablo en tierra de Alba:

«Con esta sequía tan larga se escae el ganado».

Por otra parte, además de los artículos remisivos, encontramos en el Vocabulario del canónigo salmantino una cierta heterogeneidad a la hora de enfrentarse a la labor definicional, algo que, evidentemente, da cuenta de que estamos ante la obra de un excelente aficionado a la dialectología, pero no de un lexicógrafo; esta heterogeneidad definicional, por otra parte, se reelabora en bastantes ocasiones en la definición académica posterior del DRAE-1925; si bien también se reproduce literalmente en otras.

No obstante, los artículos del Vocabulario tienen casi siempre una estructura definida que consta de lema (ej. Abalar), categoría gramatical, definición — cada acepción se separa con doble plica, pero no se numera—, localización diatópica, ejemplo de uso autoridad o ejemplo inventado- $\mathrm{y}$, en algunas ocasiones, etimología en párrafo aparte y con el encabezado «ETIM.».

\section{LA MICROESTRUCTURA DEL VOCABULARIO}

Todas las informaciones incluidas en los artículos se estructuran en el repertorio de Lamano y Beneite de diversas formas; no obstante, abordaré especialmente el tema de la localización dialectal, así como del uso actual de una determinada voz en territorio salmantino, es decir, mostrando - como pretende el canónigo salmantino - «el dialecto salmantino tal como hoy se habla» (Lamano y Beneite 1915: 5), así como la forma en que autoriza dicha voz, dado que son los aspectos que atañen especialmente a mi investigación.

\subsection{Localización dialectal}

Con respecto a la localización de voces en determinadas localidades salmantinas, Lamano y Beneite se acoge a determinadas fórmulas que, como he indicado al principio de este capítulo, suelen ser del tipo «Dícese en», «Empléase en»o «Úsase en». No obstante, utiliza diversas variantes de estas fórmulas, así como otras expresiones, para localizar sus acepciones. A continuación, reproduzco estas fórmulas con indicación de algunas entradas en las que Lamano las emplea, siempre tras la definición -0 , en su caso, remisión - oportuna:

«Acostumbran a decir este vocablo en» (encalcar, enconioso) 
«Aplican este calificativo los ribereños a los aldeanos del campo de la provincia de Salamanca, como significando que habitan éstos entre 'jaras o jarales'» (jariego)

«Corren ambos términos en la Sierra de Béjar y de Francia» (frejoni)

«Corre esta palabra en» (arrañar, baldaga, comparante, chafusca)

«Corren con frecuencia ambos términos en la comarca de Vitigudino» (esturullar) ${ }^{6}$

«Corren estas dos palabras» (arricángel, arricángele)

«Corre esta voz en» (arreponcio, escobera)

«Corre este vocablo en» (carcabonera, estrumpido, farallo, farfallo, fedegar, floriana, lana, manojar)

«Corre este término en» (balumbio, biesa, contraforaño, enjalmar, escanjillarse)

«Corren estos dos términos en la Sierra de Francia» (furaco)

«Dícese esta palabra en» (atruendo, cardeña)

«Dícese este vocablo en» (cerrebojar, corcho)

«Dícese este término en» (arboleda, cepera)

«Dícese en tierra de» (comestible, estópor, extramoniar)

«Dicen así en la Sierra de Francia» (bollagarín) ${ }^{7}$

«Dicen esta palabra en» (belfa, blanquillo, brigada)

«Dicen este término en la Sierra de Francia» (cabilio)

«Dicen este vocablo en» (brátano, búrbura, cabril, callada, cogortijo)

«Dicen esta voz en» (cance, cefear)

«Emplean esta palabra en» (bisarna, capillo, despiojar)

«Emplean este vocablo en» (boco, candín, crestar, eco, emparecer, empún, escaecer, espinzonar)

«Emplean este término en» (cancillera, capatraz, cartapela, enguerado, da, escarrabioso, sa)

«Emplean esta voz en» (bonina, corre, desamolgazar)

«Empléase es término en» (adrar, aentar, fámulo)

«En el partido de Béjar» (avión, aceituna)

«Está en uso en» (discreción (a), enchocar, escalecer)

«Es acostumbrado este término en» (bango (quedar en), cachazo)

«Es usado este término en» (atortar)

«Es de frecuente uso en» (engurrirse, entear, entuñarse, eschoncar, estofarse)

«Es de frecuente uso este vocablo en» (esborrezar, estrupio, fungar, gálbana)

«Es de muy frecuente uso en» (ázare, envencijar, gingrar)

«Es de uso frecuente en» (envivecer, entrampallar)

«Es de corriente uso en» (desencasado, da, despargillar, enguisgar,enial, espetellar)

«Es de uso corriente en» (chancero, ra, emboyar)

«Es de uso corriente este vocablo en» (estrilis)

«Es muy usado en dialecto» (delantre, enrebujar)

«Es muy usado este término en» (encobijar, faraño)

\footnotetext{
${ }^{6}$ Esta fórmula se emplea únicamente en esturullar, voz en la que se hace una remisión a esturar, después de la cual se introduce este comentario.

${ }^{7}$ En los casos en los que la fórmula se registra en el Vocabulario tan solo en una ocasión, figurará como ejemplo la entrada en la que aparece, con la referencia completa - como en el caso de bollagarín-, incluida la zona en la que la documenta Lamano y Beneite.
} 
«Es término acostumbrado en el partido de Ciudad Rodrigo» (escamoche)

«Está en uso este vocablo en» (baciero, baluquear, esganchar)

«Está en uso esta voz en» (badarras, enfatar)

«Está en uso esta palabra en las comarcas de Ciudad Rodrigo y Vitigudino» (cuartal)

«Está en uso corriente en tierra de Vitigudino» (empurrar)

«Está en uso este término en» (desvahir, esbocarado, da, escurrimbe)

«Forma usual en la Ribera del Duero» (abaivus)

«Llámanlo así en» (boil, borreciégano, cambizo, engañote, enjimbre, erreal, estrapullo)

«Llámanla así en» (bollagra, cebolluna (aguja), correcaminos)

«Llámase así en» (aguchadera, labresto)

«Lo/ la llaman así en» (bucín, caricol, codal, cuturía)

«Suele decirse este vocablo en la Sierra de Francia» (encanchalarse)

«Son frecuentes ambos vocablos, en sus varias acepciones, en la Sierra de Francia» (fagina fachina).

«Usan este vocablo en» (arruma arrutar, aseñallar, briñuelo, campina, cañajeja)

«Usan esta voz en la comarca de Ledesma» (charramudarse)

«Úsanlo en esta significación en el partido de Ciudad Rodrigo» (arrumaco)

«Usan este término en» (barde, borde, empapiñare, engiba)

«Usan estos dos vocablos en la Sierra de Francia» (frangir frangear)

«Usan esta palabra en» (barandel, cangar, empullar, engriero)

«Úsanse estos dos vocablos en los pueblos fronterizos con Portugal» (fayal)

«Úsase este vocablo en» (canchera, estrambanguión, fulanario)

«Úsase este vocablo, con ambas significaciones, en pueblos de Ciudad Rodrigo» (franja)

«Úsase esta palabra en» (arrayada, cañiceras (botas))

«Úsase este modismo dialectal en tierra de Ciudad Rodrigo» (fósfora (estar en))

«Úsase promiscuamente este vocablo, en ambos géneros, en tierra de Ciudad Rodrigo» (guácharo).

En algunas ocasiones incluye comentarios sobre la localización de dicha voz dentro de la definición:

guinda 'maroma de que se valen en los arribes del Duero para pasar el río'.

jejo 'crestón de cuarcita que hay en los cerros pizarrosos de los arribes del Duero'.

\subsection{Comentarios sobre uso / poco uso}

Lamano y Beneite emplea múltiples fórmulas para justificar la presencia de determinada voz en su repertorio; de hecho, según argumenta el propio autor al comienzo de la Introducción de El dialecto vulgar salmantino: «La presente monografía tiene por objeto estudiar "las variedades modernas, así de gramática como de vocabulario, que ofrece la lengua castellana"8 en la provincia de Salamanca» (ibid: 5).

${ }^{8} \mathrm{El}$ autor enmarca esta frase entre comillas para demostrar que su trabajo se ajusta a la convocatoria de la RAE, publicada en La Gaceta (17/10/1908), que reza exactamente: «Estudio de las variedades antiguas ó modernas, ya de gramática, ya de vocabulario, que ofrece la lengua castellana en alguna de las regiones donde se habla». Sobre este asunto véase Muriano Rodríguez (2002: 943). 
A continuación reproduzco —en orden alfabético - las frases más recurrentes a lo largo del Vocabulario, con las que Lamano y Beneite pretende demostrar el hecho de que las voces por él registradas son «variedades modernas», esto es, en uso en Salamanca:

«Como quiera que sea, es lo cierto que todas estas significaciones dialectales son de muy frecuente uso» (malvar)

«Es de muy frecuente uso» (asimesmo, cércene)

«Es de muy frecuente uso con la antítesis vocalaria» (mormullo)

«Es de muy frecuente uso dialectal este término, pero se emplea restringiéndolo a significar solamente el nacimiento de las semillas» (nacencia)

«Es frecuente oir» (cutio (de))

«Es muy frecuente decir» (dominguillo, embocar, esgalamido)

«Es muy acostumbrado este término con la antítesis vocalaria» (estucia)

«Es muy usado este término con la antítesis vocalaria» (fición)

«Es muy usado este término con la metátesis consonaria» (nesecidad)

«Es muy usado, en dialecto, con la acostumbrada epéntesis dialectal» (morchuelo)

«Es muy usado este término entre la gente aldeana» (ciégano)

«Es frase muy usada» (guindar : «iQuién te guindó!»)

Por otra parte, justifica también la actualidad de algunas voces con frases en las que resalta el hecho de que no son anticuadas, es decir, que no son arcaísmos en «dialecto salamanquino»:

«Aun se emplea, y con frecuencia, este vocablo» (arbolecer)

«Es muy usado el modismo dialectal esmorriñarse de risa, que vale tanto como morirse de risa» (esmorriñarse)

«No es anticuada esta voz adverbial, antes su uso es tan frecuente como debió de serlo en el siglo XV» (abasto)

«No es anticuado este término; antes, el vocablo culto «alfarero», es completamente desconocido por el vulgo salmanquino» (cantarero)

«No es anticuado este término en el dialecto vulgar salamanquino» (dentecer)

«No es anticuado este término, antes es muy frecuente decir» (andulencias)

«No es anticuado este término, antes bien es de muy frecuente uso» (enfastiar)

«No ha caído en desuso, antes se emplea harto» (asín)

«No ha caído en desuso este vocablo» (alardear)

«No es anticuado este término en el género masculino, sino muy usual» (calavero)

«No es anticuado este término en el dialecto salmantino» (istial)

«No es anticuado, sino de muy frecuente uso» (alargas, cachar)

«No es anticuado este término» (acuitarse, cortina)

«No es anticuado este término, sino de muy frecuente uso» (enderezo)

«No es anticuado este término, sino de muy frecuente uso dialectal» (enviejar)

Incluso combina el comentario sobre uso con la notación dialectal:

«No es anticuado este término, sino de muy frecuente uso particularmente en la Ribera del Duero» (finiquitar).

«No es anticuado este término dialectal, antes de muy frecuente uso, particularmente en la comarca de Alba de Tormes» (hongoso). 
No obstante, también emplea algunas fórmulas para introducir comentarios sobre el 'poco uso' de alguna de las voces incluidas en el repertorio, lo cual aporta a su trabajo una perspectiva histórica de la lengua que encaja a la perfección con la reproducción de textos antiguos, como el Fuero de Salamanca, en la Antología que constituye la segunda parte de su obra 9 . Así, he encontrado comentarios del tipo:

«Apenas si se usa más que en el siguiente adagio» (espigarzo)

«Apito, m. V. Apitijo. Emplean ambos vocablos indistintamente, aunque hay que reconocer que este último va cayendo en desuso» (apito).

«Apenas si se usa ya en esta acepción en que la empleó el poeta dialectal [Torres Villarroel]» (borro)

«En dialecto antiguo también se usó [...] hoy poco frecuente» (recadar)

«Es de uso poco frecuente» (cantista)

«Es hoy de muy escaso uso, aunque antiguamente debió de emplearse con frecuencia» (enjorginar)

«Es muy poco usado este término dialectal» (maralla)

«Forma enteramente desusada sin el arrimo del pronombre personal» (abá, primer lema del vocabulario, con remisión a abar)

«No se usa apenas, más que en el refrán «Conde, condidura y cebada para la mula»» (condidura)

«Va cayendo en desuso esta palabra» (aflecho)

\subsection{Autoridades ${ }^{10}$}

Con respecto a la autorización de las voces, encontramos en el Vocabulario del canónigo salmantino un amplio abanico de ejemplos entre los que cabe destacar el uso de fuentes escritas - especialmente literarias - De hecho, no es este un caso aislado en el ámbito de la lexicografía regional, pues, como indica Ahumada Lara (2003: 61):

Junto a las fuentes orales, en esto del acopio de regionalismos, las fuentes escritas (lingüísticas y metalingüísticas) han sido objeto de permanente atención tanto por la lexicografía general como por la regional; es más, las fuentes escritas han constituido históricamente, en especial para la lexicografía regional, una de sus más viejas aspiraciones.

Asimismo, el profesor Ahumada precisa aún más este juicio en un trabajo posterior, en el que concluye que:

Si bien es cierto que los repertorios de regionalismos peninsulares proliferan hasta la saciedad a lo largo del siglo XX con muy desigual valor, no lo es menos que todos aquellos que han

\footnotetext{
${ }^{9}$ Por lo que respecta al Fuero de Salamanca, a él dedica el capítulo I de la citada Antología (ibid: 69-72) e indica, en nota a pie de página que: «Tómanse los párrafos aquí trascritos, de la edición publicada por la Excelentísima Diputación de Salamanca. Madrid. Imprenta de Aribau y C., 1877» (ibid.: 69).

${ }^{10}$ Incluyo en este subapartado únicamente las fuentes escritas (lexicográficas, lingüísticas, literarias, etc.) y las fuentes orales que emplea el canónigo salmantino para autorizar sus voces; si bien debo indicar las constantes referencias, especialmente en el apartado etimológico, al Manual elemental de gramática histórica (1903) de Ramón Menéndez Pidal. De hecho, introduce comentarios como el de cansio: «En el castellano antiguo se halla también este término pero sin la $i$ epentética dialectal, y en la misma forma en que lo pronuncian los judíos de Oriente, conforme al testimonio del Sr. Menéndez Pidal (Gram. Hist. Esp., § 121)»; tras estas palabras reproduce la autoridad de Vida de Santo Domingo de Silos, de Berceo (s. v. cansío).
} 
merecido la atención de la crítica especializada hicieron de la cita literaria uno de sus principales objetivos (Ahumada Lara, 2004: 58).

En el caso de Lamano las autoridades empleadas van desde el Fuero de Salamanca hasta las obras de los «escritores dialectales contemporáneos» (Lamano y Beneite, 1915: 33) ${ }^{11}$, especialmente Luis Maldonado y Gabriel y Galán (ibid: $33-34)^{12}$, sin obviar, claro está, a los que el canónigo denomina a lo largo de toda la obra «poetas salmantinos del Renacimiento» (Juan del Encina y Lucas Fernández) ${ }^{13}$, o a Diego de Torres Villarroel ${ }^{14}$. También se halla entre los textos empleados como autoridad por Lamano y Beneite el Libro de Alexandre (s. v. bufa, perda, lamber, rachizo, relampear $)^{15}$, así como las referencias a Gonzalo de Berceo (s. v. cencío, cimajada, escogencioso, inclín), Lope de Vega —ejemplos de Las

\footnotetext{
${ }^{11}$ Recordemos que El dialecto vulgar salmantino está estructurado en tres partes: Notas gramaticales, Antología y el Vocabulario, del que aquí me ocupo. Es importante señalar que en la Antología, Lamano y Beneite recoge fragmentos que van cronológicamente desde el Fuero de Salamanca a la poesía

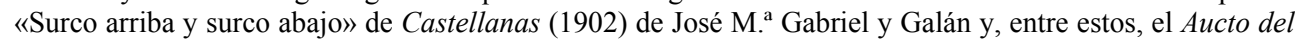
Repelón, de Juan del Encina, la Comedia hecha por Lucas Fernández, El Entremés del Villano, de Sebastián de Horozco, el Acto II de Las Batuecas del Duque de Alba, de Lope de Vega, los actos II y III de La Peña de Francia, de Tirso de Molina, las Redondillas de Manuel de Herrera Gallinato, la Glosa de la Madre Isabel de San Felipe, el Romance aldeano de Diego de Torres Villarroel, Un juicio de conciliación, de Manuel González Moro y los actos II y III de La Montaraza de Olmeda, de su contemporáneo Luis Maldonado. El canónigo salmantino justifica esta selección textual, que abarca varios siglos de historia de la lengua, porque reconoce en el dialecto salmantino la capacidad de mantener «el vetusto vocablo junto a la palabra juvenil» (id.). Así pues:

Este tan raro fenómeno podrá el lector admirarlo a su placer cuando, al repasar las hojas de la Antología, aspire el campestre aroma de las siemprevivas dialectales que vienen floreciendo, cada Mayo, desde los tiempos ya remotos del FUERO DE SALAMANCA, hasta estos días novísimos en que el inolvidable Gabriel y Galán esparció por ambos mundos sus primeras CAMPESINAS. En las páginas de la Antología puede seguirse, paso a paso, el desenvolvimiento del dialecto vulgar salmantino (ibid: 6-7).

${ }^{12}$ El canónigo salmantino no duda en decir que «entre los escritores dialectales modernos, indiscutiblemente es Luis Maldonado quien, con más feliz acierto, ha cultivado la literatura regional salmantina» (ibid: 33 ). No obstante, no concede ningún rigor dialectal al Vocabulario que este incluye al final de Del campo y de la ciudad del que dice que «si se va a decir verdad, es pobrísimo y casi no valía la pena haberlo hecho» (ibid.: 34). Así pues, concede gran valor dialectal a la obra de Luis Maldonado, pero pone en entredicho sus cualidades como dialectólogo. Asimismo, con respecto a la obra de Gabriel y Galán, no duda en señalar que sus Extremeñas (1902), «debieran servir de auténtico documento dialectal salmantino, toda vez que el lenguaje, que emplea en estas poesías, es, con muy escasas variantes, el habla de los pueblos fronterizos de las sierras de Béjar y de Francia» (id.).

${ }^{13}$ Dedica el apartado III de la Introducción (9-12) a Juan del Encina del que no duda en sentenciar que: «La literatura genuinamente dialectal salmantina comienza en las Églogas y Representaciones del que ha sido llamado "padre y fundador del teatro castellano"» (ibid.: 9). Asimismo, continúa en el apartado IV con una breve valoración de las Farsas y Églogas de Lucas Fernández (ibid.: 12-13), y llega a concluir que hay pasajes en ellas «que le granjean un salmantinismo harto más castizo que al poeta del CANCIONERO», esto es, al propio Juan del Encina.

${ }^{14}$ A él dedica el apartado XI de la Introducción (ibid.: 28-31) y emite el siguiente juicio:

Bueno es confesar [...] que pocos han reunido más ventajosas condiciones, ni se han encontrado en circunstancias más propicias para familiarizarse con el dialectismo salamanqués, como don Diego de Torres. Su temperamento literario, intensamente ironizador, su intento de observación sagacísima y nunca saciada, y su alma democrática, que diríamos hoy, le encarrilaron por las sendas tan apacibles del fol-korismo regional. ¡De aquí que el dialecto vulgar le fuera tan querido! (ibid.: 29).

${ }^{15}$ Indico únicamente algunos ejemplos de los artículos que incluyen cada autoridad aquí mencionada.
} 
Batuecas del Duque de Alba $(1638)^{16}$ _ Tirso de Molina o Miguel de Cervantes. Encontramos, por tanto; comentarios como los siguientes, junto con su correspondiente cita:

«En esta acepción se halla empleada en el Fuero de Salamanca, y es uno de los pocos términos dialectales que allí se hallan» (malvar 'inculpar')

«En este sentido la empleó también Cervantes» (atestar 'rellenar las cubas de vino cuando cesa la fermentación del mosto') ${ }^{17}$

«Fué término éste usado también por nuestros clásicos» (malencónico)

«Los poetas dialectales salmantinos la emplearon con suma amplitud [...]» $(\text { nacencia })^{18}$.

«Se ve empleado por los poetas salmantinos del Renacimiento» (albondo)

Asimismo, son autoridades recurrentes el Vocabulario de refranes y frases proverbiales (1627) de Gonzalo Correas ${ }^{19}$ o el primer diccionario monolingüe del español, el Tesoro de la lengua castellana (1611), de Sebastián de Covarrubias ${ }^{20}$; a ellos dedica las siguientes palabras en la Introducción de su obra: «Bien merecen figurar en estas páginas, como cultivadores que fueron del dialecto salamanqués los nombres, por demás ilustres, de Covarrubias y de Correas» (Lamano y Beneite, 1915: 27).

No obstante, Lamano acude en algunas ocasiones a autoridades lingüísticas como Juan de Valdés, cuyo Diálogo de la lengua emplea (s. v. emprestar 'prestar'):

La rustiquez de este vocablo no impidió el estar muy en uso en el renacimiento, según se desprende del testimonio de Valdés:

«Marcio. Unas veces siento decir prestar y otras emprestar; ¿cuál tenéis por mejor?

Valdés. Tengo por grosero el emprestar» ${ }^{21}$.

También s. v. encentar 'principiar' comenta:

\footnotetext{
${ }^{16}$ En las voces encobijar, enfición, espritu y fondo utiliza textos de esta pieza teatral de Lope; de hecho, a él dedica el apartado VI de la Introducción (ibid: 15-17) y a Tirso de Molina el VII (17-20). De ambos comenta:

Dos excelsos dramaturgos, que, en nuestro siglo de oro, dijérase que fueron el luminare majus y el luminare minus del Teatro español—Lope de Vega y Tirso de Molina—, llevaron a la escena patria asuntos salamanquinos — de carácter histórico - que justamente pasaron en una de las comarcas más típicas de esta región: en la Sierra de Francia (ibid.: 15).

No obstante, no concede el mismo valor a la utilización del «dialecto salamanquino» (ibid:: 17) en los dos dramaturgos; así pues, a juicio de Lamano y Beneite, «ese dialectismo serrano, que Tirso llevó a su Comedia Famosa, es prueba evidente de que Tirso lo aprendió, no de vista, sino de oídas, y en el mismo lugar donde pasa la escena, según puede fácilmente colegirse» (id.), mientras que «Lope habló de las Batuecas y de Miranda, no más que de oídas: de lo que oyó en el palacio ducal de Alba a los que eran entonces señores de los Estados o feudos de la serranía de Francia» (ibid: 16).

${ }^{17}$ Emplea en esta ocasión un ejemplo de Rinconete y Cortadillo: «No tardará mucho a venir Silbatillo con la canasta de color, atestada de lo que Dios ha sido servido».

${ }^{18}$ Reproduce un fragmento de las Farsas y Églogas, que incluye también en la Antología: «Digo ya, pues su nacencia / para que sean desposados, / fué tan buena y los sus hados / yo de aquí les doy licencia».

${ }^{19}$ Así, s. v. esquilar, farinato, favo, hinchir, humero, jera, mangajón, mantiniente (a), melecina, serano, solombría o tajadero, en las que se incluye la cita correspondiente del Vocabulario de Correas.

${ }^{20}$ Son ejemplos agraciar —en ese caso solo con la referencia «V. Covarrubias»—, arrollar, atañer, bastón, borborito, brebajo o cerristopa.

${ }^{21}$ Toma todos los ejemplos del Diálogo de la lengua de Orígenes de la lengua española (1737), donde Gregorio Mayans y Siscar dio a conocer la obra de Juan de Valdés.
} 
En esta acepción es de antiguo uso. Decía ya Juan de Valdés:

«Más me contenta decir embarazado que embazado, y más tardar que engorrar, y más partir que encentar».

Otro caso es el de escomenzar 'comenzar', pues, a pesar de justificar esta forma verbal con el comentario «es muy usado con la próstesis silábica», el canónigo salmantino cita a Valdés para señalar que:

Ya, en sus días de pleno y ardoroso Renacimiento clásico, recomendaba Valdés la elisión de la silaba prostética:

«Y por la mesma causa debéis quitar un es de algunos vocablos, como son: estropezar y escomenzar».

Llegados a este punto cobra especial trascendencia indicar también «lo que falta» en la bibliografía manejada por Lamano y Beneite, pues, como ya se ha indicado, la comisión encargada de conceder el premio no halla «esta memoria al corriente de la bibliografía del asunto que trata»; de hecho, por ejemplo, cabe reseñar que en la Tabla alfabética de los escritores citados en este libro (ibid.: 674), incluye dieciséis referencias a Ramón Menéndez Pidal, si bien catorce de ellas son remisiones al Manual elemental de gramática histórica (1903) (s. v. abodigar, aborrir, acerrojar, acogollar, aina, aitar, amajadalar, asestar, biforo, bimbre, cansío, ceriondo, estonces y estrébedes). No obstante, tratándose de una obra sobre el habla salmantina, área de influencia del leonés, tan solo he localizado dos remisiones a El dialecto leonés (1906): en la voz jarina, de la que indica su uso en la Ribera del Duero, y en burdio, a: «Burdo. Dícese en Tierra de Ciudad Rodrigo»», si bien Menéndez Pidal anota esta epéntesis de - $i$ en Lumbrales, en la comarca del Abadengo, al noroeste de la provincia de Salamanca (1906: 152)».

Por otra parte, las fuentes orales también están presentes en el Vocabulario, ya sea con ejemplos que el autor califica como «canción popular», «copla popular», «dicho decidero», «frase familiar», «refrán», «romance popular», como con ejemplos sin referencia y que parecen deberse a lo que el canónigo salmantino dice haber escuchado ${ }^{22} \mathrm{o}$ que, simplemente, podrían haber sido creados por él para que no falte en ninguna de sus voces la autoridad correspondiente.

Veamos algunos ejemplos cuya fuente no menciona, sino que introduce con frases del tipo «es frecuente oír», «es muy frecuente decir», etc.:

cutio (de), adv. De quieto, de asiento. Dícese en la Sierra de Béjar, no de los trabajadores temporeros, sino de los que se contratan para servir por un año o por un plazo más largo y de tiempo indefinido. Es frecuente oir:

«¿Estás de cutio o de emprestao?».

desbruciarse, r. Caerse de bruces. No es anticuada, sino de uso muy frecuente la acepción metafórica «enfermar, agravarse la enfermedad, morirse por momentos»:

«-Tiene un entreensí, un entreensí que se desbrucia, señor méico».

${ }^{22}$ En la voz pechil introduce el siguiente comentario: «En dialecto salmantino úsase, con suma frecuencia, en la Sierra y en la Ribera del Duero. Porque a más de valor dialectal lo tiene también folk-lórico, me place trascribir la siguiente frase, tomada de un diálogo entre mujeres ribereñas: «Que pa qué te digo que no quites la llave, por drento de la puerta? Pus porque las malas [las brujas, quería decir], suelen entrar por el agujero del pechil». 
embocar [...] Marchar lejos. Es muy frecuente decir:

«Embocó donde Cristo dio las tres voces».

En otros ejemplos, Lamano y Beneite introduce la denominación «canción popular» (s. v. chanca, enrejado, fato, mangajón — con la especificación «canción popular, de cuna»-riberano o serano ); «copla popular» (s. v. huertero, donde hay una «copla popular burlesca», rodeo, etc. $)^{23}$; «dicho decidero» $(\text { s. v. engüerar })^{24}$; «frase familiar»:

ñarras (a) adv. Trabajosamente. Es frase familiar:

«Andamos así, a ñarras».

peonada, f. Surcada larga.

«Buena peonada echó.»

Frase familiar con que se designa el mal suceso que ha tenido determinado negocio. Es muy usado este término entre cavariles y segadores.

Incluye también las denominaciones «romance popular» (s. v. cavaril, encordar), «refrán» (s. v. apitar, escorrozo, esturado, froncia, hacienda, humero, lamber, machorra, pernicote, vedar) o «adagio» (s. v. arraclán, hacienda, lamber —en estas dos últimas voces como sinónimo de refrán).

Finalmente, y para completar este análisis, es importante poner en valor que el Vocabulario no es solo un repertorio lexicográfico dialectal de la provincia de Salamanca, sino una muestra del español del primer cuarto del siglo XX. Así, en el primer apartado de su obra - Notas gramaticales - realiza una declaración de principios en cuanto al léxico estudiado y anota que:

Así en las NOTAS GRAMATICALES como en el VOCABULARIO van incluídas formas y palabras, no en gran copia, que no son de origen y uso exclusivo del dialecto de la provincia de Salamanca, sino que son corrientes y molientes, a todo ruedo, en el lenguaje vulgar de otras regiones. No ignorando esta particularidad, me ha parecido oportuno anotarlas, así en la Gramática como en el Vocabulario, pensando en que, si por dicha nuestra llega la hora tan ensoñada de levantar el grandioso alcázar de la Plangotología castellana, el sabio artífice que dé cima a tan bizarra empresa, cuente con que estas y aquellas determinadas voces del lenguaje vulgar leonés, pongo por caso, son también muy usadas en el lenguaje vulgar salmantino (Lamano y Beneite, 1915: 42; la cursiva es suya).

Nótese especialmente que Lamano justifica con sus palabras la inclusión de arcaísmos, vulgarismos, así como de léxico usado también en otras regiones de España, a partir de una perspectiva histórica de la lengua, en concreto del dialecto leonés, con la intención de que conste que en tierras de Salamanca también se emplean dichas voces. Así pues, a pesar de las carencias científicas que también se han puesto de manifiesto en este análisis macro y microestructural de su Vocabulario, pesan más sus aciertos, que lo convierten, sin lugar a dudas, en un referente para el estudio del español del primer cuarto del siglo XX.

\footnotetext{
${ }^{23}$ En la entrada tranlarear 'tararear' reproduce una «copla popular de cuna»: «Sólo porque te duermas, / tengo de tranlarearte, / hijo en la cuna, / cara de luna».

${ }^{24}$ Emplea esta denominación solo una vez más (s. v. juerta 'huerta'): "“No hay atajo sin trabajo, ni juerta sin arrodeo". Es dicho decidero, muy vulgar y acostumbrado en la Sierra de Francia».
} 


\section{REFERENCIAS BIBLIOGRÁFICAS}

AHUMADA LARA, Ignacio (2003): «Tradición y actualidad en la lexicografía sobre los regionalismos del español: las fuentes escritas», en M. ${ }^{a}$ Teresa Echenique Elizondo y Juan Sánchez Méndez. coords., Lexicografía y lexicología en Europa y América. Homenaje a Günther Haensch, Madrid, Gredos, pp. 61-75.

(2004): «El valor de las fuentes escritas en la lexicografía regional: Fernán Caballero y su interés para la lexicografía histórica andaluza», en Ignacio Ahumada, ed., Lexicografía regional del español, VI Seminario de Lexicografia Hispánica, Jaén, Universidad de Jaén, pp. 57-82.

DAVIES, Mark: Corpus del español (CdE): <http://www.corpusdelespanol.org/>, Universidad de Brigham Young.

FERNÁNDEZ DE GATA Y GALACHE, Manuel (1903): «Vocabulario charruno», en Ociosidades, Salamanca, Imp. y Lib. de Francisco Núñez, pp. 70-104.

LAMANO Y BENEITE, José de (1915): El dialecto vulgar salmantino, Salamanca, Tipografía Popular (Imp. de «El Salmantino») [facsímil en Salamanca, Ediciones de la Diputación de Salamanca, 2002, con pról. de Antonio Llorente Maldonado de Guevara].

Llorente MALDONAdo DE GUEVARA, Antonio (1947): Estudio sobre el habla de la Ribera (comarca salmantina ribereña del Duero), Salamanca, CSIC. [En línea: http://bibliotecadigital.jcyl.es/i18n/consulta/registro.cmd?id=636].

Muriano RodríGuEZ, M. ${ }^{a}$ Montserrat (1997-1998): «La aportación de Lamano al Diccionario de la Academia», Revista de Lexicografia, IV, pp. 137-148.

- (2001): «La incorporación de información dialectal salmantina en la historia del Diccionario académico: la decimoquinta edición», en M. Maquieira et al., eds., Actas del Segundo Congreso de la Sociedad Española de Historiografia Lingüística, León, Universidad de León, 2-5 de marzo de 1999, Arco Libros, pp. 725-735.

- (2002a): «La marca 'Sal.' (Salamanca) en los Ficheros de la Real Academia Española», en M. Campos Souto y J. I. Pérez Pascual, eds., De historia de la lexicografia, Noia, Toxosoutos, pp. 169-179.

(2002b): «El certamen de la Academia 'Estudio de las variedades antiguas ó modernas, ya de gramática, ya de vocabulario, que ofrece la lengua castellana en alguna de las regiones donde se habla' y su reflejo en el Diccionario», en M. A. Esparza Torres et al., eds., Estudios de historiografia lingüistica ofrecidos a Hans-Joshef Niederehe. Actas del III Congreso Internacional de Historiografia Lingüistica, Hamburgo, Buske, 2002, 2, pp. 943-950.

- (2012) : «La lexicografía dialectal: el papel de José Lamano y Beneite en el panorama hispánico», en Antoni Nomdedeu Rull, Esther Forgas Berdet y Maria Bargalló Escrivà (eds.), Avances de lexicografia hispánica, Tarragona, Publicaciones URV, I, pp. 441-453.

Real ACAdemia EsPañola: Banco de datos (CORDE). Corpus Diacrónico del Español [en línea]: $<$ http://corpus.rae.es/cordenet.html $>$.

- (2001): Nuevo Tesoro Lexicográfico de la Lengua Española [DVD-ROM], Madrid, Espasa-Calpe.

SÁNCHEZ LEÓN, Cándido (1995): Palabras y expresiones usadas en la provincia de Salaman$c a$, rev., reelab., ed. e introd. a cargo de Antonio Llorente Maldonado de Guevara, Salamanca, Caja Salamanca y Soria. 


\section{$\mathrm{ANEXO}^{25}$}

abá (abar)

abaivus (abar)

abaleadera (abaliadera)

abarcucia (abarcuza)

abigero (abigedo)

abinadura (abinada)

abintestate (abentestate)

abollágara (abogalla)

abollar (abollecer)

abreciar (brezar)

abrizar (brizar)

abuquerar (aburacar)

acambizar (cambizar)

acantear (acantacear)

acarabarse (acarbarse)

acarrarse (acarrearse)

aceda (acea)

aceitunera (aceituna)

acetunero (acetunera)

aciburrio (aciborrio)

acipurrio (aciburrio)

acistar (acistrar)

acomuelgar (acogolmar)

acordis (acorde)

acucioso, sa (cuicioso)

acuellar (acollar)

agaón (agadón)

agollar (abollecer)

agualija (aguadije)

aguarde (aguardo)

agüedije (aguadija)

aguijar (jijear)

aicar (latir)

ajijear (jijear)

ajujar (jijear)

ajundear (ajundiar)

alambrear (alambrar)

albentestate (abentestate)

albera (albero)

albintestate (abentestate)

albondara (albondare)

alborozar (albarazar)

alboreada (alborada)

algállara (abogalla)

aljamarero, ra (alfamarero)

amarizarse (marizarse)

amenudear (amenudar)

amial (henazo)

andancia (andancio)

\author{
andróminos (andapadres) ${ }^{26}$ \\ anialarse (anidalarse) \\ aniazo (henazo) \\ antejuela (dentejuela) \\ anusgarse (añusgarse) \\ añidiar (anidiar) \\ añodro, dra (anudrido) \\ añosgarse (añusgarse) \\ añudrirse (anudrirse) \\ añuzgarse (añusgarse) \\ apañadera (cambiza) \\ apazconar (apasconar) \\ aperchar (apechar) \\ apesgar (empesgar) \\ apiparar (apipar) \\ apito (apitijo) \\ aproveñar (probeñar) \\ arapéndale (alpéndare) \\ arbolada (alborada) \\ arrecádale (arrecádele) \\ arrejar (enrejar) \\ arresayo (arrasayo) \\ arribe (arribadero) \\ arricángele (arricángel) \\ arrimadero (arrimacho) \\ arrumiaco (arrumaco) \\ artuña (ortuña) \\ asarrayo (arrasayo) \\ atarra (atajarria) \\ atarrasca (atarracar) \\ atarre (atarra) \\ atiño, $\tilde{a} a$ (ataril) \\ atoñar (atollar) \\ aturniar (aturnear) \\ aturrear (aturnear) \\ avientar (aventar) \\ bagaña (baga) \\ barañar (abarañar) \\ bardasco (bardasca) \\ barijonda (barihonda) \\ barraco (berraco) \\ berrenda (berrendo) \\ berreondo, da (verriondo) \\ bocin (bocal) \\ bollagra (bogalla) \\ bonino (bonina) \\ boraco (buraco) \\ bornear (borlear) \\ borracete (borrecete)
}

borrajada (borrajera)
borrezo (borrecete)
borrezón (borrez)
brebajo (berberajo)
brécul (brecoles)
brécule (brécul)
brizar (brezar)
brumar (brumir)
bucheta (burcheta)
cachonera (cachón)
cagorzo (cahozo)
cahorzo (cahozo)
calabuezo (calabozo)
calagozo (calabozo)
calbochera (calboche)
calbochero (calboche)
callaza (callada)
calpuchero (calboche)
candonguear (camandulear)
caneco (caneca)
cantera (canchera)
cañaleja (cañajeja)
capacina (capaceta)
capaza (capaceta)
caramonjo (caramojo)
caramozo (caramojo)
carbizal (carbal)
carbochada (calbochada)
carbochar (calbochar)
carbochero (calbochero)
carniculas (carnícoles)
carnizón (carnazón)
cartapelo (cartapel)
cenceñada (cencellada)
cerondo, da (ceriondo)
cerrajina (cerracina)
cimarada (cimajada)
cimbrar (cimblar)
clibés (cribés)
cogolmo (cogüelmo)
comprisión (complisión)
comuelgo (cogüelmo)
conducho (condumio)
confrañimiento (confrañeo)
confrañijo (confrañeo)
confrañirse (confrañarse)
cornijal (cornejal)
correcaminos (correcamina) 


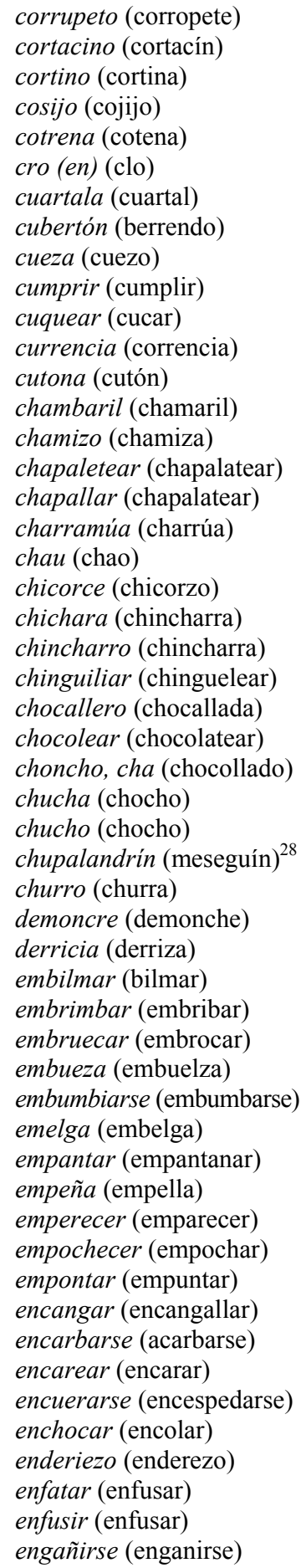

engarrañirse (engarañarse) engolondrinarse (engolondrarse)

engorra (angorra)

engorriar (engurriar)

engorro (engarrio)

enguantada (engualtada)

enguileño, $\tilde{a} a$ (enguileno)

enguitar (engaritar)

engurrubiñarse (engara-

ñarse)

enjabelgar (enjabalgar)

enjalmar (ensalmar)

enjoimbre (enjimbre)

enjumbre (enjimbre)

enquellotrar (enquillotrar)

entallicar (entallecer)

entejuela (dentejuela)

entremiso (entremijo)

entretiño (entresijo)

entruchilar (entruchar)

envair (envaer)

esbagar (esbagañar)

esbarrancar (descorcharse)

esbarrundar (esbarrumbar)

esborcillar (esborcellar)

esbruciar (desbruciar)

escaer (escaecer)

escaldunciar (escalduciar)

escampar (alambrar)

escanjarillarse (desgua-

dramillarse)

escanjillarse (esguadrami-

llarse)

escaramonjo (escaramojo)

escarcear (escarciar)

escariarse (escarearse)

escarocho (caroncho)

escarramancharse (escarnacharse)

escarrancharse (escarramancharse)

escarrapicharse (escarramancharse)

escarrollar (escaronllar)

escascamondarse (escarcadarse)

escascarillarse (escasca-

mondarse)

escavar (descavar)

escoldar (acoldarse) escombarcado,da (descombarcado)

escuajarnigar (esguadrami-

llar)

escupiñitir (escupiñitar)

escurrimbe (escurridura)

eschangarillarse (escanja-

riarse)

esfardarse (esbalugarse)

esfrañarse (confrañarse)

esguadarmillar (esgua-

dramillar)

esjambrado, da (esjambiado) esjambriado, da (esjambiado)

eslabazar (escalduciar)

esmatacanar (desmatacar)

esmiejar (esmicar)

espapar (espachurrar)

esparegir (espargir)

espeltre (espelde)

esperruchar (espachurrar)

espetellar (espetar)

estalaise (estalaís)

estempanar (estampanar)

estitero (estitadero)

estojoso, sa (estojado).

estrambanguión (cambalud)

estreldes (estrébedes)

estruncar (estroncar)

esturullar (esturar)

esturrullar (esturar)

fagina (fachina)

fantesioso, sa (fantástico)

faraño, ña (fariño)

finiquitizar (finiquitar)

finiticar (finiquitar)

fisgueo (fisgonería)

frangir (frangear)

frejoni (frejón)

friolla (frijuela)

furaco (buraco)

gabarrio (gabarro)

gabejón (gadejón)

gañivete (canivete)

garañuela (engarañado)

garbancera (gabancera)

garrobanza (garrobaza)

gasnatazo (gasnatón)

gaspazo (capaceta)

gausán (bausán)

gengibón (gengiba) 
gerrén (herrén)

gerrenar (herrenar)

gingrir (gingrar)

granio (graneo)

grumara (brumar)

grumir (brumir)

guadrimalla (guadramalla)

guañino (guadañino)

guay (guaño)

güerear (güerar)

guirre (guirle)

guirri (guirre)

guirrio (guirle)

hebarse (ahibarse)

hibado, da (ahibado)

hibarse (ahibarse)

higarado (ahigarado)

hilado (hiladillo)

hilancia (hilaza)

hojaranzal (hojaranza)

horcón (horco)

hurganero (hurgandero)

husmo (husmeo)

inas (ainas)

indiestro (inciesto)

inhiesto (indiestro)

inhiestro (indiestro)

injiestro (indiestro)

inter (inte)

intriquidante (intercadente)

iteres $y$ véntiles (íteles $\mathrm{y}$

véntiles)

jabar (abar)

jaquetonazo (jaque)

jaramandero,ra (jaramen-

dado)

jaro, ra (jaramendado)

jastial (hastial)

jerrén (herrén)

jerrenar (herrenar)

jidiguera (jediguera)

jijar (jijear)

jincarrona (jinca)

jollo (jolio)

juguera (gota)

juimbre (jumbre)

jumbrio (jumbre)

juñir (juñar)

laberiento (labariento)

laboría (laboreo)

lambrón, na (lambrión)

leenda (leyenda) légrimo, ma (lígrimo)

lígrime (lígrimo)

limio (limo)

lindón (lindero)

liño (linio)

liornia (liorna)

lubriosco, ca (lobriosco)

lucera (clarera)

lleldo, da (yeldo)

llosa (josa)

lludo, da (yeldo)

magaza (magarza)

malengrinar (malangrinar)

malinconía (malenconía)

malingrinar (malangrinar)

malinsosis (malensosis)

malóbrago (malóbado)

malvasar (malvar)

mamona (lechetrezna)

mancollera (mancolla)

mandable (mandible)

manicio (esqueje)

mañizo (manicio)

marahojo (marabaja)

marajo (maraojo)

maromo (marmelo)

marón (maroto)

marzal (marcial)

masque (manque)

mecanche (macanche)

mecido (berberajo)

medianil (medianía)

melo (mela)

melocadia (meleocadia)

mellizar (amellizar)

menducio (mundicio)

mesón (malmuza)

mezucón (mezuca)

moflear (mofear)

mojacilla (moacilla)

mondicio (mundicio)

morecer (marizar)

morgaño, ña (musgaño)

morquera (morguera)

morrarse (amorrarse)

moscalindrón (moscón)

mosquilón (moscón)

motril (moscón)

mudalar (mudadal)

muezca (muesca)

muezcar (muescar)

muezcla (muesca) muñir (mullicar)

musgaño, ña (monsigato)

nabresto (nabestro)

nubriosco, ca (lobriosco)

ondi (onde)

pago (bago)

paloteado (paleo)

palla (lapa)

papera (baluga)

papurrina (baburrina)

patijuelo (paticuelo)

patidifuso,sa (patidifuso) ${ }^{29}$

pedernio, ía (pederne)

peleles (mendos)

pelinchir (perinchir [sic])

perchar (pechar)

perrinchir (perhinchir)

piensar (apiensar)

piesgo (pielgo)

piezgo (pielgo)

pinguijar (pintear)

piojarero (pegujalero)

piojarrero (piojarero)

pipajo, ja (pipo)

pipar (apipar)

pirrarse (pilfarse)

pirro (canga)

prebe (pebre)

rabán (rabadán)

rachizar (rachar)

raldear (raldar)

ralladera (ralla)

raspalejón (raspajilón)

rebracejío (bracejío)

recadar (arrecadar)

recalcación (recalcadura)

recebollar (recebojar)

recienzo (rencencio)

rechinadero (rachisol)

rechisol (rachisol)

redil (redero)

regancho (regancha)

rejigear (jigear)

rejigeo (jigeo)

religado (lóbado)

relóbado (lóbado)

remudear (remudar)

reondir (redundir)

repipión (repimpión)

requerenciar (remudiar)

restriegar (restregar)

reundir (rejundir) 


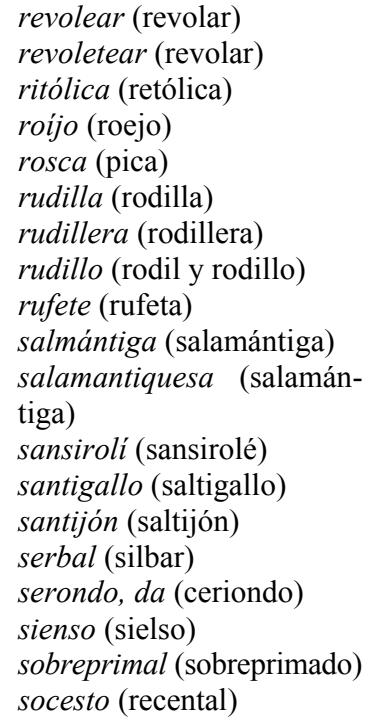

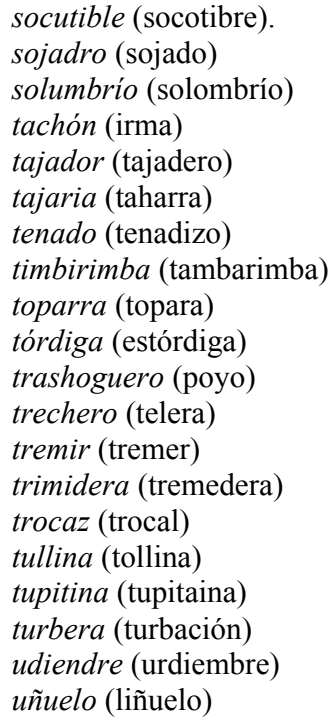

\author{
vado (vadera) \\ vafear (vadear) \\ vajear (vadear) \\ valona (baluga) \\ velambres (velaciones) \\ verdulago (verdolaga) \\ verrondio, ia (verreondo) \\ vilesa (vilera) \\ voleo (volea) \\ zalagre (zalacre) \\ zamarrón (zagarrón [sic]) \\ zangaño (zangaburra) \\ zarajuelle (zaragüelle) \\ zarraplastear (zarrapas- \\ trear) \\ zeacina (ciacilla) \\ zorollo, lla (cerezón) \\ zubicar (zahumar) \\ zumbiar (zumbar) \\ zumo (zugo) ${ }^{30}$
}

${ }^{25}$ En cuanto a la disposición de las entradas de este Anexo, incluyo el lema al que remite, entre paréntesis y en redonda; de este modo se puede comprobar que en la mayoría de las ocasiones la entrada es una variante ortográfica o fonética de aquella a la que remite.

${ }^{26}$ La entrada andapadres no se registra en el Vocabulario, pero sí en el de Fernández de Gata y Galache (1903, s. v. anda-padres) con el significado de 'pingajos'.

${ }^{27}$ Hay una errata, pues la entrada a la que debe remitir es brécoles.

${ }^{28}$ No incluye esta entrada en el vocabulario.

${ }^{29} \mathrm{Se}$ trata de una errata, pues esta entrada aparece dos veces, la primera en la página 565 con la definición 'atónito, pasmado' y una segunda en la siguiente con la remisión. Por otra parte, patidifuso no es, en absoluto, una palabra exclusivamente salmantina. De hecho, entra en el DRAE-1899 con remisión a la segunda acepción de patitieso 'que se queda sorprendido por la novedad ó extrañeza que le causa una cosa'. Puede añadirse, además, que la consulta del CORDE [25/05/12] aporta como primer testimonio de patidifuso una carta de Juan Valera a Marcelino Menéndez Pelayo, fechada en 1882: «De aquí en los poetas líricos o épicos del día no sé qué delirios malsanos, no sé qué frenético víctorhuguismo, que me deja patidifuso y me hace reír y apesadumbrarme a la vez». Sin embargo, en la consulta del $C d E$ [25/05/12] se documenta patidifusa un poco antes, ya en 1864, en la obra Del dicho al hecho, del dramaturgo Manuel Tamayo y Baus: «Bien me maliciaba yo que el buen viejo haría algo por ti. Pero, ¿quién se había de figurar?... Mira..., mira... Esta se ha quedado patidifusa».

${ }^{30}$ Se trata de un caso de circularidad, dado que zumo remite a zugo y éste último remite nuevamente a zumo. 